\section{Successful Treatment of Behcet's Eye Disease with Infliximab Therapy}

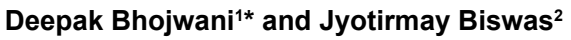 \\ ${ }^{1}$ Vitreo-Retina Consulatant, Raghudeep Eye Hospital, Ahmedabad, India \\ ${ }^{2}$ Director of Uveitis and Ophthalmic Pathology, Sankara Nethralaya, \\ Chennai, India
}

\begin{abstract}
Behcet's disease is a multisystem chronic relapsing inflammatory vasculitides. Ocular Behcet's is the most frequent cause of morbidity accounting for $25 \%$ blindness. Exact aetiology of Behcet's is unknown. Tumour Necrosis Factor (TNF) is thought to be the key mediator of inflammation. Immunosuppressive agents frequently fail to control inflammation or maintain remissions. Biological agents like infliximab have been reported to successfully induce regression of all symptoms of behcet's disease. We describe a case of Behcet's disease treated with infliximab therapy. The patient is a 15 year old boy with recurrent oral and genital ulcerations, gastrointestinal illness and uveitis. We treated him with immunosuppressive agents followed by Infliximab therapy. Infliximab therapy successfully suppressed Behcet's eye disease and also no recurrence in extraocular features was seen during our short term treatment evaluation. To our knowledge, there has not been any previously documented case report on treatment of Behcet's eye disease with Infliximab therapy from India.
\end{abstract}

Keywords: Behcet's Disease; Biological Immunomodulation; Infliximab Therapy; Tumour Necrosis Factor Blocker

\section{Introduction}

Behçet's disease is a multisystem chronic relapsing inflammatory disease classified among the vasculitides. The diagnosis of Behcet's disease is based on identification of its typical clinical features and diagnostic criteria have been laid down by the International Study Group (ISG) [1]. Ocular disease is the most frequent cause of morbidity leading to blindness in $25 \%$ of those affected [2]. The aetiology of Behçet's disease is unknown, however the current thought is that the Tumour Necrosis Factor (TNF) plays a central role in the

*Corresponding author: Deepak Bhojwani, Vitreo-Retina Consulatant, Raghudeep Eye Hospital, Ahmedabad, India, Tel: +91 7940400909; E-mail: bhojwani.deepak1986@gmail.com

Citation: Deepak B, Jyotirmay B (2015) Successful Treatment of Behcet's Eye Disease with Infliximab Therapy. J Ophthalmic Clin Res 2: 006.

Received: January 06, 2015; Accepted: January 28, 2015; Published: February 11,2015 pathogenesis of the inflammatory process [3,4]. The various immunosuppressive drugs, used either alone or in combinations frequently fail to control inflammation or maintain remissions. Various case reports have documented the role of biological immunomodulator agents like infliximab in inducing short-term remission of all manifestations of the disease, including acute sight threatening panuveitis [4].

We describe our experience with infliximab therapy in Behcet's disease in our below case report of a 15 year old boy treated at our institute.

\section{Case Description}

A 15 year old boy presented with blurring of vision in left eye since 1 month. No ocular complaints in his right eye. He gave past history of recurrent oral apthous ulcers, genital ulcers and gastrointestinal symptoms since last 3 years. Paediatric gastroenterologist had diagnosed him with Behcet's disease and referred to us for management of his ocular symptoms. Tuberculosis and paediatric connective tissue disorders like rheumatoid arthritis and ankylosing spondylitis were all excluded by relevant standard laboratory investigations by the treating gastroenterologist and rheumatologist. On examination his best corrected visual acuity was 20/30 in both eyes. Intra Ocular Pressure (IOP) was $12 \mathrm{~mm} \mathrm{Hg}$ in both eyes. Anterior segment biomicroscopic examination of his left eye revealed small keratic precipitates on inferior half of cornea with anterior chamber $2+$ cells and $2+$ flare. Right eye was quiet. Fundus examination disclosed 2+ vitritis in his left eye (Figure 1). Fundus Fluoroscein Angiography revealed widespread capillaropathy suggestive of inflammatory involvement of both arteries and veins in both eyes (Figure 2). OCT documented normal foveal architecture in both eyes (Figure 3). His systemic laboratory investigations were unremarkable including HLA- B51, HLA- B27, and Mantoux test along with Chest X-ray (tuberculin test), anti-toxoplasmosis immunoglobulins with standard systemic laboratory investigations and so we started his treatment with oral cyclosporine along with full dose systemic steroids. Topical steroids and mydriatics were also added to control anterior segment inflammation in left eye.
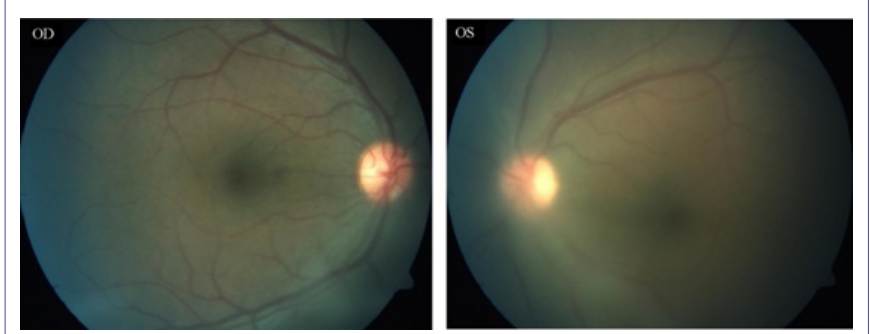

Figure 1: Posterior pole colour fundus photograph of both eyes. Note prominent Vitreous Haze (+2) evident in left eye. 

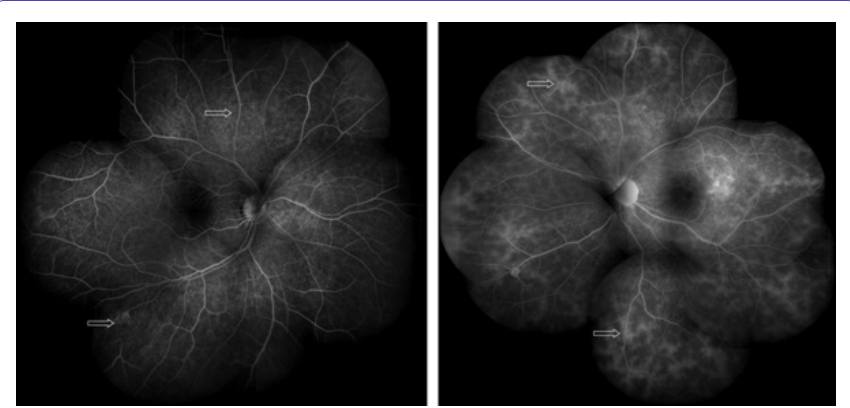

Figure 2: Fundus fluoroscein montage photograph of both eyes revealing widespread inflammatory capillary involvement (capillaropathy).

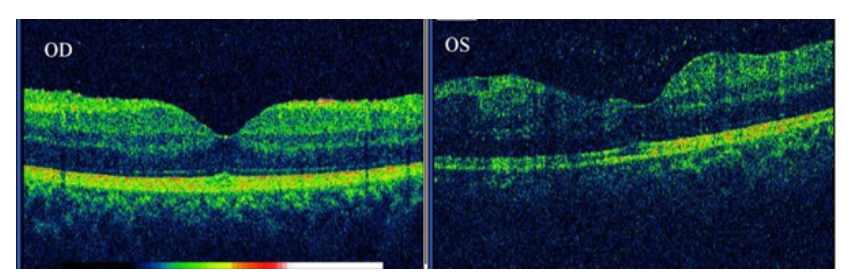

Figure 3: Spectral domain OCT documenting normal foveal architecture in right eye. Central foveal thickness OD-163 microns, and mild retinal in right eye. Central foveal thickness
thickening of 172 microns in left eye.

On second month follow-up examination, the visual acuity improved in both eyes to 20/20. Inflammation was controlled in both eyes. The patient did not experience any reoccurrence of gastrointestinal illness or oral ulcers. However his intraocular pressure was high in both eyes (OD-30 $\mathrm{mmHg}$; OS-38 $\mathrm{mmHg}$ ). Steroid response was suspected as the cause for it and the patient was prescribed antiglaucoma medications (oral acetazolamide along with dorzolamide and combigan eye drops in both eyes). We tapered down and stopped systemic steroids. Cyclosporine immunosuppressive therapy with renal function monitoring was continued.

At 6 month follow-up examination, IOP in his left eye was $22 \mathrm{~mm}$ $\mathrm{Hg}$ (still on higher side) and vitreous haze was also persisting, and so we adviced biological immunomodulator therapy with infliximab for this patient. He received infliximab infusion $5 \mathrm{mg} / \mathrm{kg}$ on day 1 , second week, sixth week and thereafter every sixth week till 6 months.

After starting systemic infliximab injections, we noted marked resolution of vitreous haze and the inflammation in both eyes has been under control. On his recent visit, visual acuity in both eyes was 20/20. IOP was $14 \mathrm{~mm} \mathrm{Hg}$ in right eye and $16 \mathrm{~mm} \mathrm{Hg}$ in left eye on topical anti-glaucoma medications. Anterior segment examination
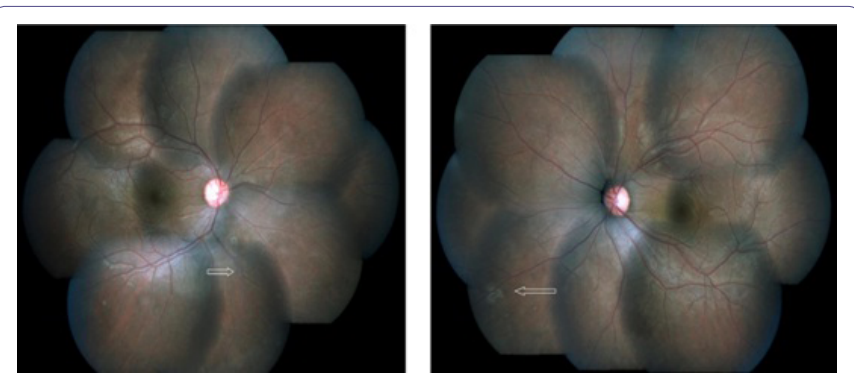

Figure 3: Spectral domain OCT documenting normal foveal architecture in right eye. Central foveal thickness OD-163 microns, and mild retinal thickening of 172 microns in left eye. of both eyes was unremarkable. Sheathing of vessels was noted on fundus examination in both eyes (Figure 4). We have followed up this patient for 1 year post infliximab therapy and there has been no recurrence of ocular or systemic disease.

\section{Discussion}

Inflammation in Behçet's disease is mediated by cytokines derived from T- helper lymphocytes type-1, including TNF [5]. Increased levels of circulating TNF and soluble TNF receptors in the peripheral blood of patients with active disease [6], as well as high levels of TNF in the aqueous humour from patients with Behçet's disease associated uveitis have been reported [7]. In vitro studies have shown that the inhibition of TNF- alpha has favourable effects on T-cell-mediated uveitis [8].

Among anti-TNF agents, infliximab and etanercept have been used in the treatment of Behçet's disease. The biggest study on interferon treatment in Behcet's disease showing the beneficial effect of interferon on eye disease was done by Demiroglu et al. Although some controversy arose previously, it was later proved that there were no ethical transgressions it that study and it was unequivocally shown that interferon alpha prevents ocular involvement in Behcet's disease [9-11]. Currently clinicians prefer Infliximab therapy over etanercept for treating Behcet's disease to avoid recurrence of the disease [12]. Data continues to accumulate suggesting infliximab administration leads to rapid and effective suppression of all Behçet's disease manifestations.[13-15] In our case infliximab therapy successfully suppressed the Behcet's eye disease during our 1 year treatment evaluation. Long term treatment outcomes with infliximab therapy are awaited. Interestingly, it also suppressed recurrence of gastrointestinal illness, oral and genital ulcerations in our case. The major constraint in starting infliximab therapy for Behcet's patients is the financial burden of treatment on patient especially in our country. Drug-induced lupus has been reported a major adverse side effect with TNF- alpha blocking therapies, including infliximab. Other rare but life threatening events reported with these therapies include infections, malignancy, heart failure, demyelinating disease [16,17]. Our patient did not suffer from any major systemic side effect during his short term infusion therapy with infliximab. We recommend monitoring of vital signs, liver function tests, tuberculin skin tests, ANA (Antinuclear Antibodies) and hemogram as a standard protocol for every patient taking TNF-alpha therapy. To our knowledge, there has not been any previously documented case report on treatment of Behcet's eye disease with Infliximab therapy from India. And so we present the following case to document regression of Behcet's eye disease with infliximab therapy.

\section{References}

1. [No authors listed] (1990) Criteria for diagnosis of Behçet's disease. International Study Group for Behçet's Disease. Lancet 335: 1078-1080.

2. Yazici H (2002) Behçet's syndrome: where do we stand? Am J Med 112: 75-76.

3. Robertson LP, Hickling $P$ (2001) Treatment of recalcitrant orogenital ulceration of Behçet's syndrome with infliximab. Rheumatology (Oxford) 40: 473474.

4. Muñoz-Fernández $S$, Hidalgo $V$, Fernández-Melón J, Schlincker $A$ Martín-Mola E (2001) Effect of infliximab on threatening panuveitis in Behçet's disease. Lancet 358: 1644.

5. Gül A (2001) Behçet's disease: an update on the pathogenesis. Clin Exp Rheumatol 19: 6-12. 
6. Evereklioglu C, Er H, Türköz Y, Cekmen M (2002) Serum levels of TNF-alpha, sIL-2R, IL-6, and IL-8 are increased and associated with elevated lipid peroxidation in patients with Behçet's disease. Mediators Inflamm 11: 87-93.

7. Santos Lacomba M, Marcos Martín C, Gallardo Galera JM, Gómez Vidal MA, Collantes Estévez E, et al. (2001) Aqueous humor and serum tumor necrosis factor-alpha in clinical uveitis. Ophthalmic Res 33: 251-255.

8. Theodossiadis PG, Markomichelakis NN, Sfikakis PP (2007) Tumor necrosis factor antagonists: preliminary evidence for an emerging approach in the treatment of ocular inflammation. Retina 27: 399-413.

9. Demiroglu H, Ozcebe OI, Barista I, Dündar S, Eldem B (2000) Interferon alfa- $2 b$, colchicine, and benzathine penicillin versus colchicine and benzathine penicillin in Behçet's disease: a randomised trial. Lancet 355: 605-609.

10. Demiroglu H (2008) Final verdict of the Turkish Supreme Courts. Br J Ophthalmol 92: 423

11. Pipitone N, Olivieri I, Salvarani C; Italian Society of Rheumatology (2012) Recommendations of the Italian Society of Rheumatology for the treatment of the primary large-vessel vasculitis with biological agents. Clin Exp Rheumatol 30: $139-161$.

12. Vitale A, Rigante D, Lucherini OM, Caso F, Muscari I, et al. (2013) Biological treatments: new weapons in the management of monogenic autoinflammatory disorders. Mediators Inflamm 2013: 939847
13. Francesco Caso, Luisa Costa, Donato Rigante, Orso Maria Lucherini, Paolo Caso, et al. (2014) Biological Treatments in Behçet's Disease: Beyond Anti-TNF Therapy. Mediators of Inflammation 2014: 1-14.

14. Maldini C, Lavalley MP, Cheminant M, de Menthon M, Mahr A (2012) Relationships of HLA-B51 or B5 genotype with Behcet's disease clinical characteristics: systematic review and meta-analyses of observational studies. Rheumatology (Oxford) 51: 887-900.

15. Cantini F, Niccoli L, Nannini C, Kaloudi O, Cassarà E, et al. Efficacy of infliximab in refractory Behçet's disease-associated and idiopathic posterior segment uveitis: a prospective, follow-up study of 50 patients. Biologics: Targets and Therapy 6: 5-12

16. Williams EL, Gadola S, Edwards CJ (2009) Anti-TNF-induced lupus. Rheumatology (Oxford) 48: 716-720.

17. De Bandt M, Sibilia J, Le Loet X, Prouzeau S, Fautrel B, et al. (2005) Systemic lupus erythematosus induced by anti-tumour necrosis factor alpha therapy: a French national survey. Arthritis Research and Therapy 7: 545-551. 